\title{
Considerações temáticas a respeito da pesquisa sobre foco na forma em inglês como L2 no Brasil
}

\author{
Ingrid Finger (FINGER, Ingrid) \\ Professora Doutora da Universidade Federal do Rio Grande do Sul - UFRGS \\ finger.ingrid@gmail.com
}

Simone Maciel Mendonça (MENDONÇA, Simone M.)

Universidade Federal do Rio Grande do Sul - UFRGS

Mestranda em Linguística Aplicada - UFRGS

mosine@ig.com.br

\section{Resumo}

Este artigo visa a apresentar um panorama referente a pesquisas realizadas nos Cursos de Pós-Graduação em Letras e Linguística no país, nas últimas duas décadas, sob a perspectiva de Foco na Forma em L2. Para tanto, foi feita uma análise dos resumos das dissertações e teses contidos no Portal da CAPES a fim de verificar os pressupostos teóricos, os tipos de procedimentos metodológicos adotados nesses estudos e os principais resultados obtidos a partir de diferentes tipos de instrução em L2. A partir da análise dos resumos coletados, foi possível perceber que os estudos se voltam para a instrução sobre gramática, instrução sobre aspectos fonético-fonológicos da L2, instrução sobre vocabulário, instrução sobre escrita, instrução sobre estratégias e instrução sobre aspectos pragmáticos da L2. Além disso, a grande maioria dos trabalhos

produzidos enfoca suas análises na instrução sobre aspectos da gramática da L2.

Palavras-Chave: Aquisição de L2, foco na forma, aquisição do inglês, instrução explícita, instrução implícita, instrução em gramática, Banco de Teses da CAPES.

\begin{abstract}
The aim of this article is to review the major research studies, carried out in Language and Linguistics Graduate Programs in Brazil, which set out to investigate the effects of focus on form instruction in English language teaching contexts in this country. The abstracts of the theses and dissertations on different types of focus on form instruction in L2, in the database Banco de Teses da CAPES, were analyzed as to the theory bases and methodologies used and main results found. The analysis showed that the studies tended to focus on instruction dealing with grammar, phonetics and phonology, vocabulary, writing, strategies, and pragmatic aspects of the L2. The great majority of the studies dealt with analyzing L2 grammar instruction.
\end{abstract}

Keywords: L2 acquisition, focus on form, English language acquisition, explicit instruction, implicit instruction, instruction in grammar, Banco de Teses da CAPES. 


\section{Introdução}

Na década de 1980, surgiu na cena mundial uma nova forma de ensino de línguas: o enfoque comunicativo. Essa nova metodologia priorizava o desenvolvimento das habilidades auditiva e oral dos aprendizes de segunda língua (L2) ${ }^{1}$ e ainda incentivava a ausência de qualquer tipo de instrução, atenção à forma da língua e ao feedback corretivo em aulas comunicativas. Essa abordagem ganhou força principalmente pela influência de duas teorias: a Hipótese do Input Compreensível (KRASHEN, 1981) e a Hipótese da Interação (LONG, 1983). Segundo Krashen (1981), é através da oferta de insumo compreensível que os alunos podem adquirir uma L2, não sendo necessário nem aconselhável que se faça qualquer tipo de ensino de gramática na sala de aula. Para Long (1983), por outro lado, são as modificações feitas durante uma conversa, modificações essas geradas pela negociação de significado por parte dos aprendizes de L2, que garantem a aquisição bem sucedida de uma língua. Para ambos os autores, portanto, não havia necessidade de instrução de gramática em ambientes comunicativos de ensino de L2.

Contudo, uma série de pesquisas realizadas ao longo das décadas de 1980 e 1990 contribuiu de maneira significativa para a descoberta de novos fatores presentes no processo de aquisição de L2, determinando os níveis de sucesso alcançados. Os resultados obtidos levaram muitos teóricos a reverem suas posições a respeito do papel do ensino de gramática em L2. Um exemplo disso aparece nos trabalhos de Long (1991, 1996), nos quais o autor faz uma revisão de sua teoria da Hipótese da Interação (1983), passando a considerar a instrução e a atenção à forma na interação conversacional como fatores importantes para que os aprendizes atinjam níveis mais elevados de sucesso.

No que diz respeito ao papel da instrução sob a perspectiva do enfoque comunicativo, Long (1991) formulou uma distinção quanto à intervenção pedagógica que pode ser realizada em um ambiente de ensino de línguas. Para ele, existem três tipos diferentes de instrução que podem ser utilizadas por um professor em uma aula comunicativa: foco nas formas (Focus on Forms), foco na forma (Focus on Form) e foco no significado (Focus on Meaning), que serão detalhadas a seguir.

A instrução sob o viés do foco nas formas dá-se quando o professor prioriza o ensino de estruturas isoladas da L2 sem que o contexto comunicativo em que tais estruturas ocorrem seja levado em consideração. Assim, para a abordagem do foco nas formas, não há uma preocupação com a relevância da ligação entre forma e significado das estruturas linguísticas em uma língua, bem como a utilidade que essas apresentam em um ato comunicativo. Essa possibilidade de abordagem de instrução limita-se ao ensino dos elementos formais da L2. Já a instrução com foco na forma preconiza que, ao serem enfatizados os elementos formais de uma língua, a atenção dos alunos de L2 volta-se à relação entre forma e significado veiculada pelas estruturas linguísticas. Ou seja, o ensino de estruturas linguísticas deve ser inserido num contexto comunicativo, no qual existe preocupação com forma, significado e uso dessas estruturas. Por outro lado, o foco no significado é a perspectiva de instrução que nega o ensino das estruturas gramaticais da L2. Segundo esse enfoque, o propósito principal de uma sala de aula de línguas deve ser a oportunidade de usar a língua em situações reais de interação. Como afirma Doughty (2001),

\footnotetext{
${ }^{1}$ Neste artigo, não será feita distinção entre os termos 'aquisição' e 'aprendizagem', nem entre as expressões ‘segunda língua (L2)’ e ‘língua estrangeira (LE)’ (ELLIS, 1994).
} 
(o) fator que consistentemente distingue o foco na forma de outros enfoques pedagógicos é o requisito de que o foco na forma leva os aprendizes a prestarem atenção (attend to) à forma, significado e uso durante um evento cognitivo, mesmo que brevemente e talvez simultaneamente. Acredita-se que esse tipo de processamento em conjunto facilita o mapeamento cognitivo entre formas, significado e uso que é fundamental à aprendizagem da linguagem. (DOUGHTY, 2001, p. 211)

Nesse sentido, dadas as três possibilidades de instrução dentro de uma sala de ensino comunicativo, pesquisas têm demonstrado efeitos positivos na adoção de atividades com foco na forma. Esse tipo de instrução conduz o aprendiz a um aperfeiçoamento tanto no seu conhecimento da L2 quanto na sua habilidade de usar esse conhecimento em atos de comunicação. Sob a perspectiva de instrução com foco na forma, por exemplo, alguns procedimentos pedagógicos têm sido adotados a fim de transformar o insumo em algo visualmente mais saliente para os aprendizes de L2, como, por exemplo, o fornecimento de feedback corretivo (recasting) ou intensificação do input (enhancement).

É grande o número de estudos investigando o foco na forma que vêm sendo realizados nos últimos anos. No Brasil, em particular, a comprovação desse fato pode ser feita através de uma consulta ao portal do Banco de Teses da CAPES (http://www.capes.gov.br/servicos/bancoteses.html), que traz resumos de dissertações e de teses defendidas no país de 1987 até 2007. Uma pesquisa rápida nas informações veiculadas nesse portal mostra um crescimento significativo no interesse de pesquisa nessa área a contar do começo da última década. Tal constatação deu origem a este artigo, que tem por objetivo mostrar um panorama das pesquisas realizadas sob a perspectiva do foco na forma em inglês como L2 nos Programas de Pós-Graduação em Letras e Linguística do país, desde 1987. O presente estudo justifica-se principalmente pela dificuldade encontrada na divulgação de informações concernente aos trabalhos realizados sob o tema foco na forma no Brasil. Além disso, é ainda pequeno o número de publicações em periódicos científicos brasileiros que relatam investigações conduzidas sobre a temática de foco na forma em L2.

A próxima seção mostra os procedimentos de coleta de dados relativos à temática da pesquisa. Na seção seguinte, são apresentados os resultados principais do levantamento. A seguir, é apresentada a discussão desses resultados sob a perspectiva do foco na forma.

\section{Procedimentos para o levantamento dos dados}

Os dados da pesquisa referentes aos trabalhos de Pós-Graduação - dissertações de Mestrado e teses de Doutorado - realizados no Brasil, tendo por enfoque o foco na forma na aquisição do inglês como L2, foram coletados no Banco de Teses da CAPES. Acessando o site dessa instituição, podemos perceber que estão disponíveis ao público apenas os resumos das dissertações e teses escritas por alunos de todo o país, sem que haja a possibilidade de acesso aos trabalhos na íntegra. É importante observar que, ao analisarmos as informações disponibilizadas no portal, notamos que existem problemas de formatação nos resumos quanto à explicitação do conteúdo dos trabalhos, assim como problemas relacionados à própria elaboração dos resumos que, muitas vezes, são incompletos ou confusos na descrição tanto de objetivos e hipóteses quanto de procedimentos metodológicos adotados. Apesar dessas restrições, fizemos um 
levantamento das produções de Mestrado e Doutorado envolvendo o tópico foco na forma, tentando verificar os aportes teóricos adotados, os objetivos e procedimentos utilizados e os principais resultados oriundos das investigações realizadas pelos estudantes brasileiros nos vários programas de Pós-Graduação em Letras e Linguística no país.

A fim de fazer o levantamento dos dados, entramos no portal da CAPES http://www.capes.gov.br/servicos/bancoteses.html e selecionamos a opção Resumos. A partir desse procedimento, nos foi possível visualizar uma caixa de diálogo, a qual permitia que se fizesse a procura das dissertações e teses por autor, assunto, instituição, nível de formação e ano de conclusão. Nossa coleta foi feita por assunto, buscando trabalhos que incluíssem as palavras-chave foco na forma, input linguístico, ensino de gramática, gramática, atenção, instrução e L2, instrução em LE, instrução explícita, instrução explícita dedutiva, instrução explícita indutiva, instrução implícita, ênfases instrucionais, processamento do input, correção de erros, feedback corretivo, estratégias de aprendizagem, papel da instrução. Nossa pesquisa também considerou os correspondentes termos em língua inglesa com o intuito de ampliar a recuperação de dados pelo sistema eletrônico.

Em um primeiro momento, o número de resultados obtidos foi bem significativo, já que foram selecionados pelo banco de dados todos os trabalhos da área de Letras, Linguística/Linguística Aplicada que continham as palavras-chave procuradas. Partimos, então, para a leitura dos títulos das produções a fim de selecionarmos as que mais se enquadravam à nossa demanda. Desse modo, acessando especificamente cada um dos trabalhos, encontramos, dentre outras informações, àquelas relativas ao nome do autor, título do trabalho, ano da produção, seguidas do nível de formação, da instituição de ensino superior, do nome do orientador e da biblioteca depositária da obra e do resumo. Se achássemos que a dissertação ou tese era pertinente à pesquisa, partíamos para a leitura do resumo. Os trabalhos que apresentaram resumos condizentes com os objetivos da pesquisa foram selecionados para uma posterior análise das informações, servindo, portanto, de base para o presente artigo. De todos os trabalhos encontrados, foram selecionadas 40 produções entre dissertações e teses. Na seção seguinte, serão apresentados os resultados do levantamento dos dados coletados.

\section{Resultados do levantamento}

Na presente seção, apresentamos os resultados do levantamento dos trabalhos sob a perspectiva do foco na forma realizado com base nas informações disponíveis ao acesso público no portal da CAPES. Primeiramente, expomos as instituições nas quais foram elaboradas as produções e ainda a ordem cronológica dos trabalhos realizados. Em seguida, abordamos as principais temáticas dos estudos encontrados pela pesquisa.

\section{Cronologia e instituições ligadas às pesquisas}

De posse dos dados, percebemos que os trabalhos escritos por estudantes brasileiros dos cursos de pós-graduação tendo por temática central o foco na forma começaram a surgir na cena brasileira na década de 1990. Conforme informações disponíveis no site da CAPES, em 1991 foi defendida a primeira dissertação sobre esse assunto na UFSC (Universidade Federal de Santa Catarina). Essa Universidade, de 
1991 a 2007, contribuiu com 5 estudos sobre o foco na forma em inglês como L2. A única instituição que a superou em número de trabalhos realizados foi a UFRGS (Universidade Federal do Rio Grande do Sul), que chegou a um total de 8 pesquisas no mesmo período. Depois dessas, temos a UFF (Universidade Federal Fluminense) e a PUC/RS (Pontifícia Universidade Católica do Estado do Rio Grande do Sul), que atingiram a soma de 4 estudos, a UNICAMP (Universidade Estadual de Campinas) e a UECE (Universidade Estadual do Ceará) com 3 estudos, a UNESP (Universidade Estadual Paulista Júlio de Mesquita Filho), a UCPEL (Universidade Católica de Pelotas), a UFG (Universidade Federal de Goiás) com 2 pesquisas cada e, por fim, a UNISINOS (Universidade do Vale do Rio dos Sinos), UFPR (Universidade Federal do Paraná), a UEL (Universidade Estadual de Londrina), a UFMG (Universidade Federal de Minas Gerais), a UNB (Universidade de Brasília), a UFPB (Universidade Federal da Paraíba), e a UFS (Universidade Federal do Sergipe) com 1 estudo produzido em cada uma dessas instituições sob a perspectiva teórica do foco na forma em inglês como L2, perfazendo um total de 40 trabalhos entre dissertações e teses escritas. Assim, notamos que 16 instituições de ensino superior desenvolveram pesquisas a respeito desse assunto no Brasil nas últimas décadas.

A seguir, a Figura 1 apresenta a distribuição dos estudos sob a temática do foco na forma realizados nas 16 instituições de ensino superior do Brasil desde a década de 1990. Nela, podemos visualizar a distribuição dos trabalhos realizados nas diferentes regiões do país sob essa perspectiva teórica. Analisando esse gráfico, podemos perceber uma concentração de estudos produzidos nas instituições de ensino superior situadas nas regiões Sul (54\%) e Sudeste (25\%) do país. No entanto, realizam-se também pesquisas nas regiões Nordeste (13\%) e Centro-Oeste (8\%), mas em menor número. A única região que não apresentou nenhuma pesquisa dentro da perspectiva do foco na forma foi a Norte.

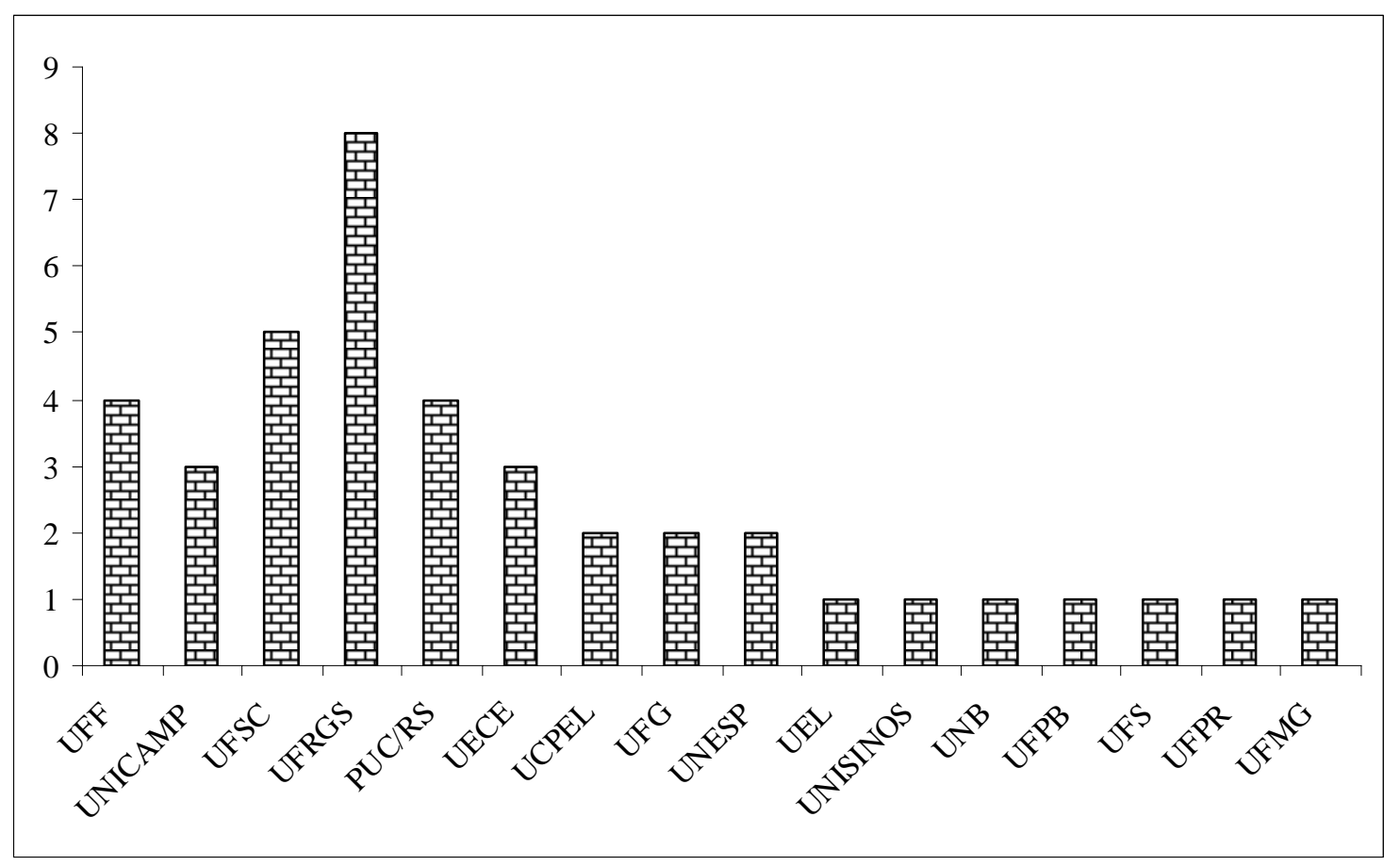

Figura 1: Distribuição das pesquisas em foco na forma por instituição de ensino superior 
Como vemos na Figura 2 abaixo, a região Sul contribuiu com 54\% (22 estudos) do total de trabalhos coletados (40) no Banco de Teses da CAPES, tendo por temática o foco da forma. Na região Sudeste houve a produção de 25\% (10) dos estudos sob essa perspectiva teórica. Além disso, na região Centro-Oeste, 3 estudos foram produzidos, perfazendo $8 \%$ do total de trabalhos sobre o assunto e, na Nordeste, 13\% (5 estudos).
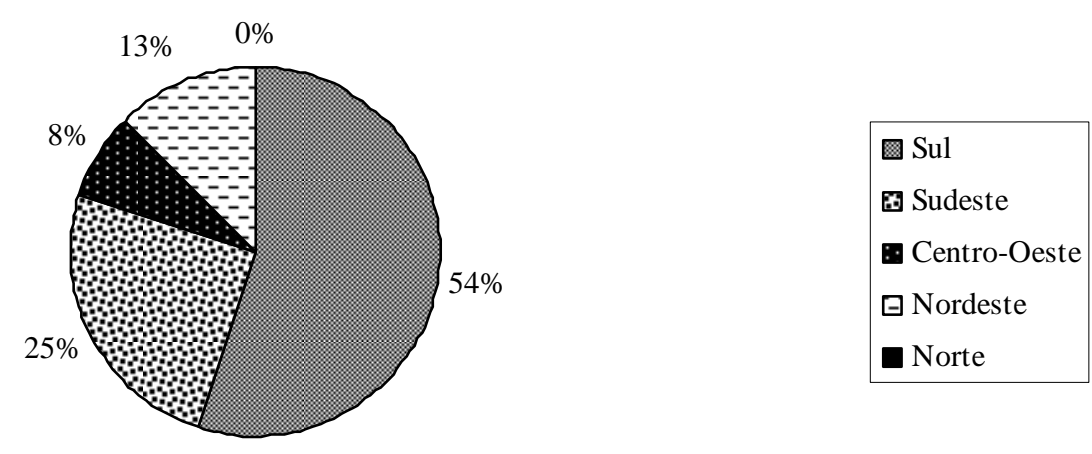

Figura 2: Distribuição percentual de trabalhos em foco na forma por região

Por outro lado, no que tange à distribuição cronológica dos trabalhos, os dados sugerem um aumento considerável do número de pesquisas realizadas no país. Os anos de 2001, 2002, 2003 e 2004 foram aqueles que tiveram uma maior ocorrência de defesas de dissertações e teses sob essa temática, cerca de 57,50\% (23) do número total de trabalhos (40). Essa evolução demonstra um aumento de interesse nesse período pela pesquisa sob a perspectiva do foco na forma pelos pós-graduandos brasileiros.

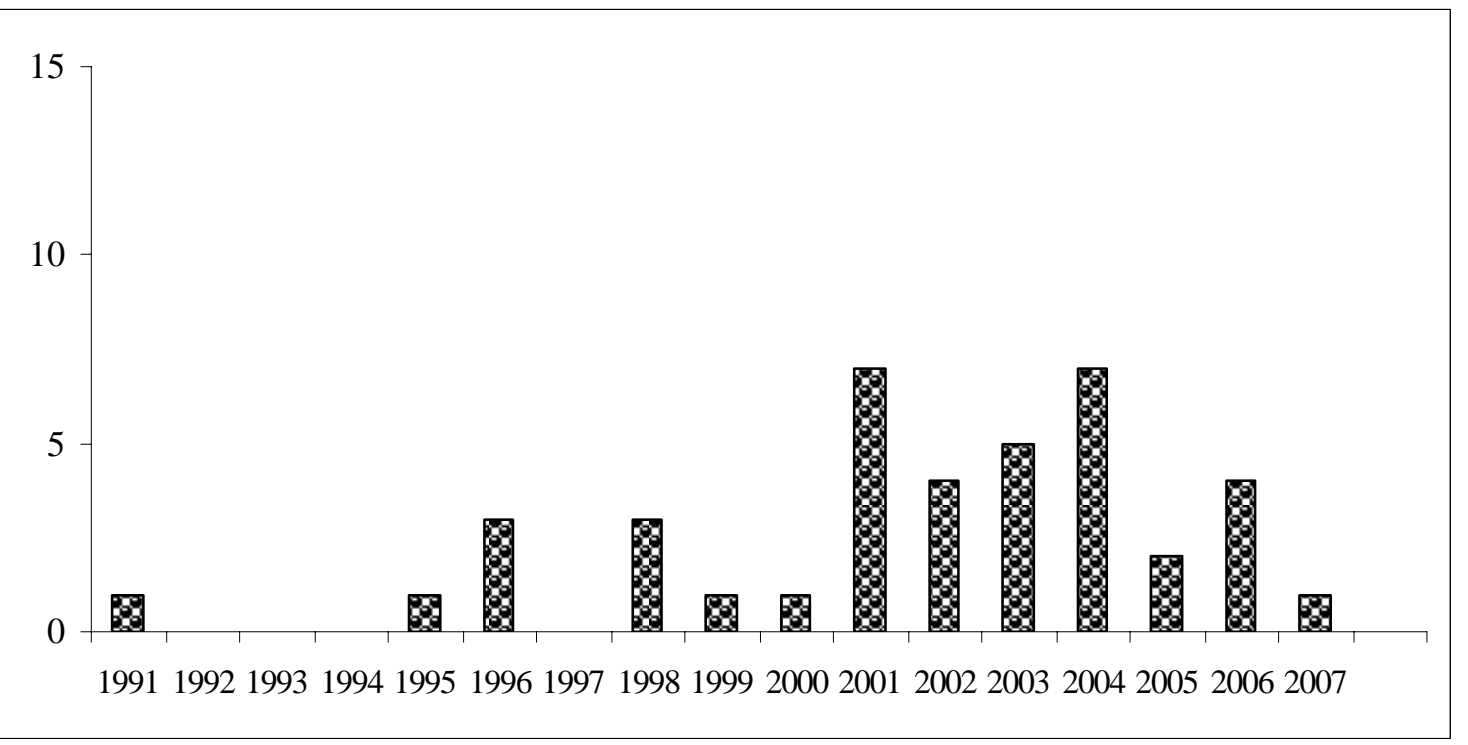

Figura 3: Distribuição cronológica das pesquisas em foco na forma 


\section{Principais temáticas de pesquisa}

Percebemos, pela leitura dos resumos das dissertações e teses envolvendo o tópico instrução em inglês como L2, encontrados no Banco de Teses da CAPES, que esses se subdividem em seis temáticas principais, a saber: (1) instrução sobre gramática, (2) instrução sobre aspectos fonético-fonológicos da L2, (3) instrução sobre vocabulário, (4) instrução sobre escrita, (5) instrução sobre estratégias, e (6) instrução sobre aspectos pragmáticos da L2. Além desses seis temas principais, durante a seleção de dados, foram também encontrados trabalhos que abordavam outros aspectos do processo de aquisição de $\mathrm{L}^{2}$. Partindo do fato de que somente as pesquisas agrupadas nos itens (1) e (2) acima (ou seja, instrução sobre gramática da L2 e instrução sobre aspectos fonético-fonológicos da L2, respectivamente) podem ser identificadas como investigações envolvendo foco na forma per se e ainda considerando que os dados referentes à instrução sobre aspectos fonético-fonológicos do inglês como L2 já foram apropriadamente investigados por Silveira e Baptista (2007), no artigo intitulado $A$ Pesquisa em Interfonologia: Percepção, Produção e Ensino, a discussão neste artigo voltar-se-á somente para os dados constantes no item 1 da classificação acima proposta. A tabela abaixo mostra tanto a classificação dos trabalhos agrupados pelos principais temas quanto os autores que os desenvolveram.

\begin{tabular}{|l|l|}
\hline \multicolumn{1}{|c|}{ Assuntos Abordados } & \multicolumn{1}{|c|}{ Autores } \\
\hline $\begin{array}{l}\text { (a) Discussão teórica sobre as } \\
\text { possibilidades de interação entre } \\
\text { conhecimento explícito e implícito a } \\
\text { partir da instrução em L2 }\end{array}$ & Ferreira (2001) \\
\hline (b) Estudos empíricos & \\
\hline $\begin{array}{l}\text { (1) Instrução sobre aspectos gramaticais } \\
\text { do inglês }\end{array}$ & \\
\hline - Formação de perguntas & Rodrigues, B. (2001) \\
\hline - Orações condicionais & Berk (1996) \\
\hline - Artigo definido & Farias (1996) \\
\hline - Formas dativas & Sousa (2006) \\
\hline - Phrasal verbs & Gontijo (2001) \\
\hline $\begin{array}{l}\text { - Processamento do input lingüístico } \\
\text { - forma e significado }\end{array}$ & Lucena (1998) \\
\hline - Ensino explícito e implícito na \\
instrução em L2 & Veiras (2003) \\
\hline $\begin{array}{l}\text { - Uso de tarefas comunicativas com } \\
\text { foco no sentido }\end{array}$ & Barbirato (1999) \\
\hline $\begin{array}{l}\text { - Papel da gramática na leitura de } \\
\text { textos }\end{array}$ & Amaral (2001) \\
\hline
\end{tabular}

\footnotetext{
${ }^{2}$ Alguns desses, entretanto, como, por exemplo, Frade (1994), Gil (1999) e Silva, S. V. (2004), que tratam de análise de crenças e de discurso do professor de inglês, não foram considerados aqui, por não se enquadrarem em nenhuma das especificações que norteiam este trabalho, pois não dizem respeito a aspectos descritivos e/ou explicativos do processo de aquisição de L2.
} 


\begin{tabular}{|l|l|}
\hline $\begin{array}{c}\text { - Proposta de metodologia de ensino } \\
\text { com Foco na Forma para o } \\
\text { desenvolvimento da expressão } \\
\text { escrita }\end{array}$ & Silva, D. (2004) \\
\hline - Feedback corretivo & $\begin{array}{l}\text { Beretta (2001); Cunha (1998); Cunha } \\
\text { (2003); Cavalari (2005); McCulloch } \\
\text { (1995); Freudenberger (2004); Menti } \\
\text { (2003); Menti (2006); Oliveira (2004); } \\
\text { Silva, M. (2005); Silva, S. C. (2004); }\end{array}$ \\
\hline $\begin{array}{l}\text { (2) Instrução sobre aspectos fonético- } \\
\text { fonológicos }\end{array}$ & * Ver Silveira e Baptista (2007) \\
\hline $\begin{array}{l}\text { (3) Instrução sobre vocabulário } \\
\text { (4) Instrução sobre escrita }\end{array}$ & $\begin{array}{l}\text { Del Valle (2004); Galvão (2002); Ferrari } \\
\text { (2002); Leite (2006); Moreira (2000); } \\
\text { Rodrigues, D. (2002); Scaffaro (2006); } \\
\text { Zilles (2001) }\end{array}$ \\
\hline $\begin{array}{l}\text { (5) Instrução sobre estratégias de } \\
\text { aprendizagem }\end{array}$ & $\begin{array}{l}\text { Rodrigues Jr. (2001); Vidal (2003); } \\
\text { Silveira (1998); Tavares (1991) }\end{array}$ \\
\hline (6) Instrução sobre aspectos & $\begin{array}{l}\text { Gadelha (2002); Giordani (2004); Gomes } \\
\text { (2003); Martins (1996); Reis (2004) }\end{array}$ \\
\hline pragmáticos da L2 & Mulling (2007) \\
\hline
\end{tabular}

Quadro 1: Classificação dos trabalhos coletados por assuntos abordados e autores

Dessa forma, notamos que, dos 40 trabalhos selecionados, 1 estudo foi feito enfocando essencialmente aspectos teóricos sobre as possibilidades de interação entre conhecimento explícito e conhecimento implícito a partir da instrução em L2, sem realizar nenhum tipo de investigação empírica (FERREIRA, 2001). ${ }^{3}$ Ou seja, a maioria dos estudos sob a perspectiva em foco na forma, encontrados no Banco de Teses da CAPES - um total de 39 trabalhos -, fez algum tipo de levantamento de dados para testar a teoria abordada ao longo da dissertação ou tese.

No que tange especificamente aos tipos de instrução, dos 39 trabalhos que tratam diretamente desse tema, 21 referem-se à instrução sobre gramática na L2 (53,85\%), 8 abordam a instrução sobre vocabulário (20,51\%), 4 voltam-se para a instrução sobre escrita $(10,26 \%), 5$ relacionam-se à instrução sobre estratégias de aprendizagem (12,82\%) e somente 1 trata de instrução sobre aspectos pragmáticos em L2 (2,56\%). No gráfico abaixo, podemos visualizar a distribuição dos tipos de instrução em relação ao número total de trabalhos relacionados diretamente a esse assunto.

Abaixo, a Figura 4 mostra a distribuição dos tipos de instrução em relação ao número total de trabalhos sob a temática do foco da forma.

\footnotetext{
${ }^{3}$ Vale lembrar que os dados relatados aqui são baseados exclusivamente na informação contida nos resumos selecionados no portal da CAPES. Assim, quaisquer imprecisões quanto aos conteúdos das dissertações e teses que possam vir a surgir ocorrem devido à nossa dificuldade de acesso a descrições mais precisas dos estudos realizados.
} 


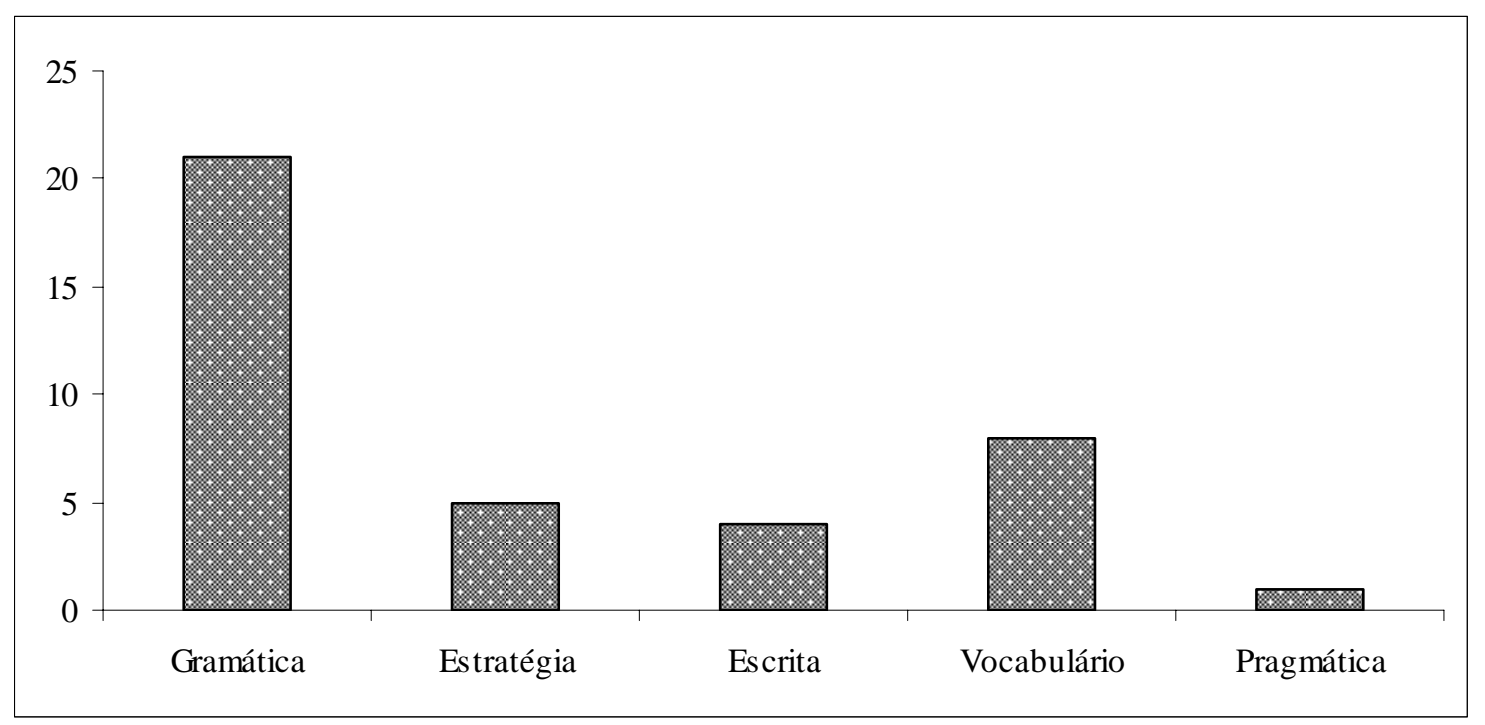

Figura 4: Tipos de instrução em relação ao total de trabalhos sob essa temática

\section{Principais resultados}

No que diz respeito à instrução sobre aspectos da gramática do inglês, os trabalhos centram as suas análises no ensino de gramática com ênfase no foco na forma em orações condicionais (BERK, 1996), na formação de perguntas em inglês (RODRIGUES, B., 2001), no uso do artigo definido (FARIAS, 1996), na alternância de formas dativas (SOUSA, 2006), em phrasal verbs (GONTIJO, 2001), no processamento da forma e do significado do input lingüístico (LUCENA, 1998), na comparação entre o ensino explícito e implícito na instrução em L2 (VEIRAS, 2003), na investigação sobre características, nos limites e possibilidades de aplicação da perspectiva do foco na forma e na proposta de metodologia de ensino com foco na forma para o desenvolvimento da expressão escrita (SILVA, D., 2004), no papel da gramática na leitura e compreensão de textos em inglês (AMARAL, 2001) e no uso de tarefas comunicativas com foco no sentido em comparação com atividades que priorizem o foco na forma (BARBIRATO, 1999).

Quanto à instrução sobre gramática em termos de feedback corretivo, os estudos selecionados abordam a instrução em foco na forma e o fornecimento de feedback (FREUDENBERGER, 2004), o tipo de feedback mais frequentemente adotado (CUNHA, 1998; CAVALARI, 2005; SILVA, M., 2005; MENTI, 2006), o feedback corretivo como gerador de output modificado (CUNHA, 2003), a influência dos dados positivos e negativos na correção de erros (McCULLOCH, 1995), o feedback corretivo nas produções escritas (BERETTA, 2001; OLIVEIRA, 2004), e a verificação do efeito do feedback corretivo dos tipos recast e elicitação (MENTI, 2003).

Os trabalhos referentes à instrução sobre estratégia, por sua vez, tratam das estratégias de comunicação através da oralidade (GIORDANI, 2004), do ensino explícito de estratégias de aprendizagem (GOMES, 2003; REIS, 2004) e do desenvolvimento de estratégias de leitura (MOREIRA, 2000; GADELHA, 2002).

No que tange à instrução sobre a escrita, os temas dos trabalhos estão relacionados à maneira através da qual os alunos revisam os textos produzidos em inglês (SILVEIRA, 1998), à compreensão e escrita de resumos por aprendizes brasileiros de inglês (TAVARES, 1991) e aos efeitos de diferentes tarefas na produção escrita com base no foco na forma (VIDAL, 2003). 
Em relação à instrução sobre vocabulário, as pesquisas abordam a instrução implícita e explícita de vocabulário (ZILLES, 2001; SCAFFARO, 2006), a instrução explícita (MOREIRA, 2000; FERRARI, 2002; GALVÃO, 2002; LEITE, 2006) e a abordagem do insumo pré-modificado, modificado e produção estimulada (DEL VALLE, 2004).

Finalmente, foi encontrado somente um estudo que testou os efeitos da instrução explícita sobre aspectos pragmáticos do inglês como L2 (MULLING, 2007).

Nesse sentido, notamos que a temática do foco na forma tem sido alvo de interesse nos estudos de Lingüística Aplicada por todo o Brasil. Entretanto, a instrução em pragmática ainda não está sendo investigada por muitos estudantes dos programas de pós-graduação das instituições de ensino superior brasileiras.

\section{Considerações finais}

Tomando-se por base os pressupostos teóricos de Long (1991) quanto aos diferentes tipos de instrução possíveis de serem adotados em uma aula comunicativa em L2, fizemos uma pesquisa no banco de dados da CAPES com o intuito de selecionarmos resumos de dissertações e teses que baseassem suas análises no emprego de instrução com foco na forma em inglês como L2 em contextos de ensino brasileiros. Assim, procuramos verificar como estão sendo estudados temas relacionados a essa perspectiva nos programas de Pós-Graduação em Letras e Linguística do país.

Pela análise dos resumos coletados no portal da CAPES, foi possível observar que os estudos se voltam para a instrução sobre gramática, instrução sobre aspectos fonético-fonológicos da L2, instrução sobre vocabulário, instrução sobre escrita, instrução sobre estratégias e instrução sobre aspectos pragmáticos da L2. Além disso, percebemos que a grande maioria dos trabalhos produzidos enfoca suas análises na instrução sobre aspectos da gramática da L2. Tal fato aponta para uma tendência aos estudos referentes à gramática no campo da linguística e da pesquisa sobre aquisição e ensino da L2. Uma questão relacionada a essa constatação diz respeito ao fato de que, quando comparada com o ensino de vocabulário, de aspectos da pronúncia ou da pragmática da L2, a instrução sobre a gramática da língua-alvo pode parecer mais propensa à reflexão consciente e à manipulação do input na sala de aula, aspectos que estão claramente presentes em situações de ensino explícito.

\section{Referências}

AMARAL, Kleny Pires do. A língua e a norma: reflexões sobre o papel da gramática da leitura e compreensão de textos em inglês. 2001. 152f. Dissertação (Mestrado em Educação) - Universidade Federal de Sergipe, Aracaju, 2001.

Banco de Teses da Coordenação de Aperfeiçoamento de Pessoal de Nível Superior CAPES. Disponível em: <http://www.capes.gov.br/servicos/bancoteses.html>. Acesso em: set. 2007.

BARBIRATO, Rita de Cássia. A tarefa como ambiente para aprender LE. 1999. 204f. Dissertação (Mestrado em Lingüística Aplicada) - Universidade Estadual de Campinas, Campinas, 1999. 
BERETTA, Juliana Maria. A correção de erros: inimiga ou aliada? A reescrita como estratégia de correção e de conscientização na produção escrita de aprendizes de inglês como LE. 2001. 130f. Dissertação (Mestrado em Letras) - Universidade Federal do Rio Grande do Sul, Porto Alegre, 2001.

BERK, Bianca Pimentel. Possible effects of input of FL learning: three distinct sets of methodological emphases for the EFL classroom learning of the conditionals. 1996. 152f. Dissertação (Mestrado em Letras) - Universidade Federal Fluminense, Niterói, 1996.

CUNHA, Ana Paula de Araújo. O tratamento de erros orais em sala de aula de inglês como língua estrangeira. 1998. 130f. Dissertação (Mestrado em Letras) - Universidade Federal do Rio Grande do Sul, Porto Alegre, 1998.

O feedback corretivo como gerador de output modificado em classes comunicativas de inglês como língua estrangeira. 2003. 279f. Tese (Doutorado em Letras) - Universidade Federal do Rio Grande do Sul, Porto Alegre, 2003.

CAVALARI, Suzi Marques Spatti. O tratamento do erro na oralidade: uma proposta focada em características da interlíngua de alunos de inglês como língua estrangeira. 2005. 194f. Dissertação (Mestrado em Estudos Lingüísticos) - Universidade Estadual Paulista Júlio de Mesquita Filho, São Paulo, 2005.

DEL VALLE, Angélica. Investigating the effects of modified input and pushed output on the comprehension and acquisition of L2 vocabulary. 2004. 98f. Dissertação (Mestrado em Letras) - Universidade Federal de Santa Catarina, Florianópolis, 2004.

DOUGHTY, Catherine. Cognitive underpinnings of focus on form. In: ROBINSON, Peter (Ed.). Cognition and second language instruction. Cambridge: Cambridge University Press, 2001.

ELLIS, Rod. The study of second language acquisition. Cambridge, UK: CUP, 1994.

FARIAS, Selma Borges Barros de. Effects of input enhancement on the EFL classroom acquisition of the definite article. 1996. 106f. Dissertação (Mestrado em Letras) Universidade Federal Fluminense, Niterói, 1996.

FERRARI, Magaly. Ampliação e reforço do vocabulário em língua estrangeira através da narração e da leitura de histórias infanto-juvenis. 2002. 121f. Dissertação (Mestrado em Lingüística e Letras) - Pontifícia Universidade Católica do Rio Grande do Sul, Porto Alegre, 2002.

FERREIRA, Herbert Luiz Braga. Gramática e aquisição: a relação entre o foco na forma e a aquisição de língua estrangeira em situação institucional. 2001. 277f. Tese (Doutorado em Lingüística Aplicada) - Universidade Estadual de Campinas, Campinas, 2001. 
FRADE, Celso. Instrução gramatical no ensino de inglês como língua estrangeira: investigação e crítica. 1994. 208f. Dissertação (Mestrado em Lingüística Aplicada) Pontifícia Universidade Católica de São Paulo, São Paulo, 1994.

FREUDENBERGER, Francieli. Instrução com foco na forma e instrução com foco no sentido: diferenciando a interação na sala de LE. 2004. 95f. Dissertação (Mestrado em Letras) - Universidade Federal do Rio Grande do Sul, Porto Alegre, 2004.

GADELHA, Isabel Maria Brasil. A leitura em língua inglesa no ensino fundamental. 2002. 274f. Tese (Doutorado em Lingüística e Letras) - Pontifícia Universidade Católica do Rio Grande do Sul, Porto Alegre, 2002.

GALVÃO, Mirian Rufini. A aprendizagem de vocabulário e a produção lexical de aprendizes de língua estrangeira: o caso do inglês. 2002. 119f. Dissertação (Mestrado em Letras) - Universidade Estadual de Londrina, Londrina, 2002.

GIL, Gloria. The metalinguistic dimensions of the foreign language classroom: discourse perspectives on focus-on-form episodes. 1999. 304f. Tese (Doutorado em Letras) - Universidade Federal de Santa Catarina, Florianópolis, 1999.

GIORDANI, Elisângela Vilela Freitas. Estratégias de aprendizagem no ensino da compreensão oral: taxonomia e instrução. 2004. 235f. Dissertação (Mestrado em Linguística Aplicada) - Universidade de Brasília, Brasília, 2004.

GOMES, Alessandra de Oliveira. O ensino explícito da estratégia de aprendizagem "agrupamento" (grouping) a alunos de inglês como língua estrangeira. 2003. $184 f$. Dissertação (Mestrado em Letras e Linguística) - Universidade Federal de Goiás, Goiânia, 2003.

GONTIJO, Elizabeth Assunção. O foco na forma de verbos frasais para aprendizes de inglês como língua estrangeira: uma nova proposta. 2001. 161f. Dissertação (Mestrado em Estudos Linguísticos) - Universidade Federal de Minas Gerais, Belo Horizonte, 2001.

KRASHEN, Stephen. Second language acquisition and second language learning. Oxford: Pergamon Press, 1981.

LEITE, Anelise de Souza. Aprendizado lexical em língua inglesa em ambiente formal de ensino por falantes do português: uma abordagem conexionista. 2006. 111f. Dissertação (Mestrado em Lingüística e Letras) - Pontifícia Universidade Católica do Rio Grande do Sul, Porto Alegre, 2006.

LONG, Michael. Native speaker/non-native-speaker conversation and the negotiation of comprehensible input. Applied Linguistics, v. 4, n. 2, p. 126-141, 1983.

Focus on form: a design feature in language teaching methodology. In: DE BOOT, Kees; GINSBERG, Ralph; KRAMSCH, Claire. (Eds.). Foreign language research in cross-cultural perspective. Amsterdam: John Benjamins, 1991. 
The role of the linguistic environment in second language acquisition. In: RITCHIE, William; BHATIA, Tej. (Eds.). Handbook of research on second language acquisition. New York: Academic, 1996.

LUCENA, Maria Inês Probst. Processing input: an investigation into Brazilian EFL students. 1998. 119f. Dissertação (Mestrado em Letras) - Universidade Federal de Santa Catarina, Florianópolis, 1998.

MARTINS, Márcia Maria de Farias Nunes. The use of learning strategies by intermediate level EFL learners when writing essays. 1996. 143f. Dissertação (Mestrado em Letras) - Universidade Federal Fluminense, Niterói, 1996.

McCULLOCH, Anne Marie Moor. A influência de dados positivos e dados negativos na aquisição de língua estrangeira. 1995. 194f. Dissertação (Mestrado em Linguística e Letras) - Pontifícia Universidade Católica do Rio Grande do Sul, Porto Alegre, 1995.

MENTI, Magali de Moraes. Efeito de dois tipos de feedback corretivo - recast $e$ elicitação - no desempenho de alunos de inglês com LE. 2003. 192f. Dissertação (Mestrado em Linguística e Letras) - Pontifícia Universidade Católica do Rio Grande do Sul, Porto Alegre, 2003.

O que norteia a escolha de professores de língua estrangeira por diferentes tipos de feedback corretivo. 2006. 179f. Tese (Doutorado em Letras) - Universidade Federal do Rio Grande do Sul, Porto Alegre, 2006.

MOREIRA, Maria Amélia Quelhas. Aquisição de vocabulário por intermédio da leitura. 2000. 103f. Dissertação (Mestrado em Letras) - Universidade Federal do Paraná, Curitiba, 2000.

MÜLLING, Lydia Tessmann. Notar e aprender: o papel da instrução explícita na aquisição da pragmática da L2. 201p. Dissertação (Mestrado em Letras) Universidade Católica de Pelotas, Pelotas, 2007.

OLIVEIRA, Marcos Nonato de. A prática do feedback no ensino da escrita em inglês como LE. 2004. 160f. Dissertação (Mestrado em Linguística Aplicada) - Universidade Estadual do Ceará, Fortaleza, 2004.

REIS, Leonice Passarella dos. Investigating the effects of language learning strategies teaching on the learning process of EFL students with learning difficulties. 2004. 102f. Dissertação (Mestrado em Letras) - Universidade Federal de Santa Catarina, Florianópolis, 2004.

RODRIGUES, Bernadette Marie da Silveira. Os estágios de formação de perguntas em inglês como língua estrangeira por aprendizes brasileiros submetidos à instrução com foco na forma. 2001. 163f. Dissertação (Mestrado em Letras) - Universidade Federal do Rio Grande do Sul, Porto Alegre, 2001.

RODRIGUES, Daniel Fernando. O ensino de vocabulário em aulas de inglês como língua estrangeira: foco na produção oral. 2002. 166f. Dissertação (Mestrado em 
Estudos Linguísticos) - Universidade Estadual Paulista Júlio de Mesquita Filho, São Paulo, 2002.

RODRIGUES JR, Jáder Martins. Processo e produto: uma avaliação do ensino da escrita em um curso básico de língua inglesa. 2001. 141f. Dissertação (Mestrado em Linguística Aplicada) - Universidade Estadual do Ceará, Fortaleza, 2001.

SCAFFARO, Andréa Peixoto. O uso da atividade de contar histórias como recurso na retenção de vocabulário novo na língua inglesa com criança na fase pré-escolar. 2006. 127p. Dissertação (Mestrado em Linguística Aplicada) - Universidade do Vale do Rio dos Sinos, São Leopoldo, 2006.

SILVA, Diana Costa Fortier. Focus on Form: integrando forma e significado no ensino da habilidade gramatical e língua estrangeira. 2004. 170f. Dissertação (Mestrado em Lingüística Aplicada) - Universidade Estadual do Ceará, Fortaleza, 2004.

SILVA, Michele Dionísio da. Correção de erros orais pelo professor em aulas de inglês: um estudo com uma turma de alunos universitários. 2005. 189f. Dissertação (Mestrado em Letras e Lingüística) - Universidade Federal de Goiás, Goiânia, 2005.

SILVA, Severino Carlos da. O processo ensino-aprendizagem de vocabulário de língua inglesa em um contexto de sala de aula. 2004. 125f. Dissertação (Mestrado em Letras) - Universidade Federal da Paraíba, João Pessoa, 2004.

SILVA, Suelene Vaz da. Crenças relacionadas à correção de erros: um estudo realizado com dois professores de escola pública e seus alunos. 2004. 176f. Dissertação (Mestrado em Letras e Linguística) - Universidade Federal de Goiás, Goiânia, 2004.

SILVEIRA, Rosane. Writing instruction and its effects on the development of EFL students' writing processes: focus on revision. 1998. 134f. Dissertação (Mestrado em Letras) - Universidade Federal de Santa Catarina, Florianópolis, 1998.

; BAPTISTA, Bárbara O. A pesquisa em Interfonologia: percepção, produção e ensino. In: IX Congresso Internacional de Fonética e Fonologia, 2006, Belo Horizonte. Anais do IX Congresso Internacional de Fonética e Fonologia. Belo Horizonte, 2007.

SOUSA, Denilson Amade. Estratégias de processamento de insumo e a alternância das formas dativas em inglês. 2006. 100f. Dissertação (Mestrado em Linguística Aplicada) - Universidade Estadual de Campinas, Campinas, 2006.

TAVARES, Roseanne Rocha. The effects of main idea identification introduction on EFL students reading comprehension and summary writing. 1991. 181f. Dissertação (Mestrado em Letras) - Universidade Federal de Santa Catarina, Florianópolis, 1991.

VEIRAS, Daniele Borchardt. As formas de conhecimento implícito e explícito na sala de aula de língua estrangeira e a formação da competência comunicativa. 2003. 142f. Dissertação (Mestrado em Letras) - Universidade Católica de Pelotas, Pelotas, 2003. 
VIDAL, Rejane Teixeira. Interlanguage stretching: the role of consciousness-raising communicative tasks and metatalk. 2003. 496f. Tese (Doutorado em Letras) Universidade Federal Fluminense, Niterói, 2003.

ZILLES, Marcelo. O ensino e a aquisição de vocabulário em contexto de instrução de língua estrangeira. 2001. 192f. Dissertação (Mestrado em Letras) - Universidade Federal do Rio Grande do Sul, Porto Alegre, 2001. 